\title{
Dependable wireless communications: applications and practices
}

\author{
Muhammad Alam ${ }^{1,2} \cdot$ Nadjib Ait Saadi ${ }^{3} \cdot$ Mian Ahmad Jan ${ }^{4} \cdot$ Xiaohua Xu ${ }^{5}$ \\ Published online: 23 June 2021 \\ ( I) Institut Mines-Télécom and Springer Nature Switzerland AG 2021
}

Over the past couple of decades, wireless communication technologies have become immensely adopted in various fields, appearing in a plethora of applications ranging from tracking victims, responders, and equipment in disaster scenarios to machine health monitoring in networked manufacturing systems, etc. Most of these applications are highly dependent on the performance of the underlying wireless communication technology. In most cases, these systems are required to have dependable timeliness requirements since data communication must be conducted within predefined temporal bounds along with fulfilling other requirements such as reliability and security. This is mainly because the unfulfillment of these requirements may compromise the expected behavior of the system and cause economic losses or endanger human lives. In addition, the broadcast nature of wireless communications in an open environment makes it more vulnerable to unwanted external entities compared to the wired communications. This makes the support of dependable wireless communications in open environments, where multiple devices are contending for the resources, a challenging task. As a consequence, future wireless communications must tackle these challenging issues such as low communication reliability, real-time support, security, reachability, and fault-tolerance. Therefore, we decided to organize a special issue focusing on this topic and to invite researchers to contribute to this research area. Overall, the response was very good. We received a total of 36 submissions among which 7 were selected for publication.

Muhammad Alam

alam@av.it.pt

1 School of Engineering, London South Bank University, London, UK

2 Instituto de Telecomunicações, Universidade de Aveiro, Aveiro, Portugal

3 UVSQ Paris-Saclay University, Paris, France

4 Abdul Wali Khan University Mardan, Mardan, Pakistan

5 Department of Computer Science, Kennesaw State University, Kennesaw, GA, USA
Hereafter, we provide a summary of each paper published in this special issue.

The first paper titled "The Robust Deep Learning Based Schemes for Intrusion Detection in Internet of Things Environments" presents a work on the security of IoT. The authors used a Fast Gradient Sign Method (FGSM) to generate adversarial samples to test the robustness of three IDS models based on Convolutional Neural Network (CNN), Long Short-Term Memory (LSTM), and Gated Recurrent Unit (GRU). They used three training methods: the first method aims to train the models with normal samples, the second to train the models directly with adversarial samples, and the last to pretrain the models with normal samples and then use adversarial samples to train the models.

The second paper titled "Design of Portable and Multifunctional Dependable Wireless Communication Platform for Smart Health Care" presents a work on the dependability aspect of wireless communication in health care systems. A portable and multifunctional Software Defined Radio (SDR) platform is designed to detect different activities of human life, especially in monitoring of health. The wireless channel state information (WCSI) in the presence of the human body is investigated to capture movements using different frequency bands. The orthogonal frequency division multiplexing (OFDM) with 64 subcarriers, magnitude, and phase response in the frequency domain is used to capture the WCSI of the activity. The design is validated through simulation and real-time experiments.

The third paper titled "A Single-Player Monte Carlo Tree Search Method Combined with Node Importance for Virtual Network Embedding" presents an algorithm that combines the basic Monte Carlo Tree Search (MCTS) method with the node importance to apply domain-specific knowledge. For a virtual network request, the authors first model the embedding process as a finite Markov Decision Process (MDP), where each virtual node is embedded in one state in the order of node importance. A shortest-path algorithm is then applied to embed links in the terminal state and return the cost as one part of the reward. 
The fourth paper titled "The Identity Authentication of WIFI System Based on Network Security" analyzes the security problems existing in current WIFI sharing applications by designing two different network authentication programs. IEEE 802.1x protocol is used for wireless access, EAP-TLS, and RADIUS bidirectional authentication mechanism to achieve the authentication of access users, and onetime password authentication technology is used in Fat AP networking mode. Aiming at the defects of the S/KEY protocol, such as server impersonation and system crash, an authentication scheme combining the DES and RSA algorithms is proposed and implemented.

The fifth paper titled "An Energy-efficient Data Aggregation Approach for Cluster-based Wireless Sensor Networks" presents a novel, lightweight, and energy-efficient functionbased data aggregation approach for a cluster-based hierarchical WSN. This approach works at two levels: the node level and the cluster head level. At the node level, the data aggregation is performed using the exponential moving average (EMA), and a threshold-based mechanism is adopted to detect any outliers to improve the accuracy of data aggregation. At the cluster head level, a modified version of the Euclidean distance function is developed to provide highly refined aggregated data to the base station.

The sixth paper describes "SOSW: Scalable and Optimal Nearsighted Location Selection for Fog Node Deployment and Routing in SDN-based Wireless Networks for IoT Systems". The SOSW is made of two parts: (i) The first one proposes to use singular-value decomposition (SVD) and QR-factorization with column pivoting linear algebra methods over the traffic matrix of the network to compute the optimum locations for fog nodes. (ii) The second one discusses a new heuristic-based traffic engineering algorithm, called Constraints-based Shortest Path Algorithm (CSPA), which uses an Ant Colony Optimization (ACO) algorithm to optimize the path computation process for tasks offloading.

The last paper titled "An Enhanced Energy Optimization Routing Protocol for WSNs" focuses on the technique of extending the network lifetime by reducing and balancing energy consumption and describes an enhanced energy optimization routing protocol (EEOPRP) for WSNs.

The Guest Editors would like to thank all the authors and reviewers for the quality of their contribution which made this special issue possible.

Publisher's note Springer Nature remains neutral with regard to jurisdictional claims in published maps and institutional affiliations. 